\title{
COMPUTATION APPROACHES FOR PARAMETER ESTIMATION OF WEIBULL DISTRIBUTION
}

\author{
Yunn-Kuang Chu and Jau-Chuan Ke
}

Department of Applied Statistics, National Taichung Institute of Technology, Taichung 404, Taiwan, R.O.C.

ykchu@ntit.edu.tw, jauchuan@ntit.edu.tw

\begin{abstract}
This paper examines the estimation comparison of two methods for Weibull parameters, one is the maximum likelihood method and the other is the least squares method. A numerical simulation study is carried out to understand performance of the two methods. Based on sample root mean square errors, we make a comparison between the two computation approaches. We find that the least squares method significantly outperforms the maximum likelihood when the sample size is small.
\end{abstract}

Key Words- Weibull distribution, Maximum likelihood method, Least squares method, Sample root mean square error, Simulation

\section{INTRODUCTION}

In the field of applied mechanics, one of distribution functions of wide applicability is the Weibull distribution. The cumulative Weibull distribution function can be given by

$$
F(x)=\left\{\begin{array}{cc}
1-e^{-\alpha x^{\beta}}, & x \geq 0 \\
0, & x<0
\end{array}\right.
$$

Its corresponding probability density is as

$$
f(x)=\left\{\begin{array}{cl}
\alpha \beta x^{\beta-1} e^{-\alpha x^{\beta}}, & x \geq 0 \\
0, & x<0
\end{array}\right.
$$

where $\alpha$ and $\beta$ are positive constants. This is the well-known "Weibull' family of distributions, named after the Swedish engineer Waloddi (1887-1979) who popularized its application for reliability analysis, especially for metallurgical failure modes.

The constant $\alpha$ is called the scale parameter, because it scales the $x$ variable. On the other hand, the constant $\beta$ is called the shape parameter, because it decides the 
shape of the rate function $R(x)=\alpha \beta x^{\beta-1}$. If $\beta$ is less than 1 the rate is decreasing with $x$. Whereas if $\beta$ is greater than 1 the rate is increasing with $x$. And if $\beta=1$ the rate is constant, in which case the Weibull distribution becomes the exponential distribution. The three different-type shapes of the rate functions for the Weibull family of distributions can be referred to Hoyland and Rausand [1]. Recently, Yang \& Nie [2], Dai et.al. [3], and Shi et. [4] presented an advanced algorithm for maximum likelihood estimation of three parameter Weibull distribution.

The maximum likelihood method and the least squares method are usually used to estimate the population parameters of a distribution. Existing in literature, computational comparison on the two estimation methods is not found. This would motivate us to study the computation approach for the Weibull parameters.

The objective of this paper is to perform a comparative efficiency on estimating parameters of the Weibull distribution between the two methods. A numerical simulation study is carried out to investigate behavior of each estimation method. Based on sample root mean square errors, we determine which method provides superior estimates of the parameters of the Weibull distribution. According to simulation results, some useful information is summarized to engineers and scientists.

\section{MAXIMUM LIKELIHOOD ESTIMATION}

Suppose that $X_{1}, X_{2}, \ldots, X_{n}$ are independent and identically distributed Weibull random variables each having probability density function $f(x)$ expressed in equation (2), where the parameters are assumed unknown. To estimate the parameters $\alpha$ and $\beta$, the maximum likelihood method is employed. The likelihood function of $X_{1}, X_{2}, \ldots, X_{n}$ can be constructed from equation (2) as

$$
L(\alpha, \beta)=\prod_{i=1}^{n} f\left(x_{i}\right)=\alpha^{n} \beta^{n}\left(\prod_{i=1}^{n} x_{i}\right)^{\beta-1} \exp \left(-\alpha \sum_{i=1}^{n} x_{i}^{\beta}\right) .
$$

Taking natural logarithmic transformation, we obtain

$$
\ln L(\alpha, \beta)=n \ln \alpha+n \ln \beta+(\beta-1) \sum_{i=1}^{n} \ln x_{i}-\alpha \sum_{i=1}^{n} x_{i}^{\beta} .
$$

Differentiating $\ln L(\alpha, \beta)$ with respect to $\alpha$ and $\beta$ respectively yields

$$
\begin{aligned}
& \frac{\partial}{\partial \alpha} \ln L(\alpha, \beta)=\frac{n}{\alpha}-\sum_{i=1}^{n} x_{i}^{\beta} \\
& \frac{\partial}{\partial \beta} \ln L(\alpha, \beta)=\frac{n}{\beta}+\sum_{i=1}^{n} \ln x_{i}-\alpha \sum_{i=1}^{n} x_{i}^{\beta} \ln x_{i}
\end{aligned}
$$


Equating to zero shows that the maximum likelihood estimates $(\hat{\alpha}, \hat{\beta})$ of $(\alpha, \beta)$ are solutions of the following equations

$$
\begin{aligned}
& \frac{n}{\hat{\alpha}}=\sum_{i=1}^{n} x_{i}^{\hat{\beta}}, \\
& \frac{n}{\hat{\beta}}+\sum_{i=1}^{n} \ln x_{i}=\hat{\alpha} \sum_{i=1}^{n} x_{i}^{\hat{\beta}} \ln x_{i},
\end{aligned}
$$

or equivalently,

$$
\begin{aligned}
& \hat{\alpha}=n / \sum_{i}^{n} x_{i}^{\hat{\beta}}, \\
& n+\hat{\beta} \sum_{i=1}^{n} \ln x_{i}=n \hat{\beta} \sum_{i=1}^{n} x_{i}^{\hat{\beta}} \ln x_{i} / \sum_{i=1}^{n} x_{i}^{\hat{\beta}} .
\end{aligned}
$$

Equations (9)-(10) can then be solved numerically for $\hat{\beta}$, which will also determine $\hat{\alpha}$ subsequently. For detailed process of the maximum likelihood method, the interested reader can refer to [5]. However, rather than pursuing the maximum likelihood method any further, let us consider a second method in the next section, which is not only computationally easier but also, as indicated by a simulation study, produces more accurate estimates of $(\alpha, \beta)$ when the sample size $n$ is small enough.

\section{LEAST SQUARES ESTIMATION}

Let $X_{1}, X_{2}, \ldots, X_{n}$ be a random sample from the Weibull distribution of equation (2). From (1), its distribution given by

$$
F(x)=1-e^{-\alpha x^{\beta}}, x \geq 0
$$

which implies

$$
\ln (1-F(x))=-\alpha x^{\beta},
$$

or

$$
\ln (1 /(1-F(x)))=\alpha x^{\beta} .
$$

Consequently

$$
\ln \ln (1 /(1-F(x)))=\beta \ln x+\ln \alpha .
$$


Now let $X_{(1)}<X_{(2)}<\ldots<X_{(n)}$ represent the order statistics of $X_{1}, X_{2}, \ldots, X_{n}$. That is,

$X_{(i)}=$ the $i$ th smallest of $X_{1}, X_{2}, \ldots, X_{n}, \quad$ for $i=1,2, \ldots, n$, and the data results in $X_{(i)}=x_{(i)}$.

If we were able to approximate the quantities $\ln \ln \left(1 /\left(1-F\left(x_{(i)}\right)\right)\right)$, we could establish the values of

$$
y_{i} \approx \beta \ln x_{(i)}+\ln \alpha, \quad i=1,2, \ldots, n
$$

Subsequently, we could choose $\alpha$ and $\beta$ to minimize the sum of the square errors. That is, $\alpha$ and $\beta$ are chosen to minimize

$$
\sum_{i=1}^{n}\left(y_{i}-\beta \ln x_{(i)}-\ln \alpha\right)^{2} .
$$

Following Hogg et al. [6] and Roussas [7], we define

$$
\begin{aligned}
& \tilde{\beta}=\frac{\sum_{i=1}^{n} y_{i} \ln x_{(i)}-n \overline{\ln x} \bar{y}}{\sum_{i=1}^{n}\left(\ln x_{(i)}\right)^{2}-n(\overline{\ln x})^{2}}, \\
& \tilde{\alpha}=e^{\bar{y}-\tilde{\beta}(\overline{\ln x})},
\end{aligned}
$$

where $\overline{\ln x}=\sum_{i=1}^{n} \ln x_{(i)} / n$ and $\bar{y}=\sum_{i=1}^{n} y_{i} / n$. We conclude that the preceding minimum (equation (13)) is attained when $(\alpha, \beta)=(\tilde{\alpha}, \tilde{\beta})$.

To apply the foregoing, we need to determine values $y_{i}$ that approximate $\ln \ln \left(1 /\left(1-F\left(x_{(i)}\right)\right)\right)=\ln \left(-\ln \left(1-F\left(x_{(i)}\right)\right)\right), \quad i=1,2, \ldots, n$. We now present one approach for doing this. Using the fact that

$$
E\left[F\left(X_{i}\right)\right]=i /(n+1) \text {. }
$$

And then approximate $F\left(x_{(i)}\right)$ by $E\left[F\left(X_{(i)}\right)\right]$. Therefore, this approach calls for using $y_{i}=\ln \left\{-\ln \left(1-E\left[F\left(X_{(i)}\right)\right]\right)\right\}=\ln \{-\ln (1-i /(n+1))\}=\ln \{-\ln [(n+1-i) /(n+1)]\}$.

Substituting these $y_{i}(i=1,2, \cdots, n)$ into equations (14) and (15), we easily obtain $\tilde{\alpha}$ and $\tilde{\beta}$. The $(\tilde{\alpha}, \tilde{\beta})$ is called the least squares estimates of $(\alpha, \beta)$.

In the next section, a numerical simulation study is conducted to understand performance of the maximum likelihood estimates $(\hat{\alpha}, \hat{\beta})$ and the least squares estimates $(\tilde{\alpha}, \tilde{\beta})$. 


\section{THE SIMULATION STUDY}

In order to investigate behavior of $(\hat{\alpha}, \hat{\beta})$ and $(\tilde{\alpha}, \tilde{\beta})$, a numerical simulation study is carried out. In the simulation experiment we set three Weibull distributions on $X$ with different combinations of parameters $(\alpha, \beta)$, in which the corresponding probability density function, distribution function, mean, variance and type of failure rate are illustrated in Table 1 below.

Table 1. Three types of Weibull distributions of $X$ used in our simulation study.

\begin{tabular}{|c|c|c|c|c|c|}
\hline$(\alpha, \beta)$ & $p . d . f$. of $X$ & $d . f$. of $X$ & $\begin{array}{c}\text { Mea } \\
\mathrm{n}\end{array}$ & $\begin{array}{c}\text { Varia } \\
\text { nce }\end{array}$ & $\begin{array}{c}\text { Type of rate } \\
\text { function }\end{array}$ \\
\hline$(1,0.5)$ & $f(x)=0.5 e^{-\sqrt{x}} / \sqrt{x}, x$ & $F(x)=\left(1-e^{-\sqrt{x}}\right) I_{(0, \infty)}$ & 2 & 20 & $\begin{array}{c}\text { Decreasing } \\
\text { rate }\end{array}$ \\
\hline$(1,1)$ & $f(x)=e^{-x}, x>0$ & $F(x)=\left(1-e^{-x}\right) I_{(0, \infty)}$ & 1 & 1 & $\begin{array}{c}\text { Constant } \\
\text { rate }\end{array}$ \\
\hline$(1,2)$ & $f(x)=2 x e^{-x^{2}}, x>0$ & $F(x)=\left(1-e^{-x^{2}}\right) I_{(0, \infty)}$ & $\sqrt{\pi} / 2$ & $1-\pi / 4$ & $\begin{array}{c}\text { Increasing } \\
\text { rate }\end{array}$ \\
\hline
\end{tabular}

For each Weibull distribution assumed on $X$, we choose a random sample $\left(x_{1}, x_{2}, \ldots, x_{n}\right)$ of size $n(=5,10,15,20,30,40,50,60,70,80,90,100)$ from $X$. Using equations (9), (10), (14), (15), and (17), we obtain the maximum likelihood estimates $(\hat{\alpha}, \hat{\beta})$ and the least squares estimates $(\tilde{\alpha}, \tilde{\beta})$ of the Weibull parameters $(\alpha, \beta)$, respectively. The above single simulation run is replicated $N=1000$ times. Hence we calculate a sequence of maximum likelihood estimates $S_{1}=\left\{\left(\hat{\alpha}_{1}, \hat{\beta}_{1}\right),\left(\hat{\alpha}_{2}, \hat{\beta}_{2}\right), \ldots,\left(\hat{\alpha}_{1000}, \hat{\beta}_{1000}\right)\right\}$ as well as a sequence of least squares estimates $S_{2}=\left\{\left(\tilde{\alpha}_{1}, \tilde{\beta}_{1}\right),\left(\tilde{\alpha}_{2}, \tilde{\beta}_{2}\right), \cdots,\left(\tilde{\alpha}_{1000}, \tilde{\beta}_{1000}\right)\right\}$. Subsequently, we compute the sample mean and the sample root mean square errors for $S_{1}$ and $S_{2}$ in terms of the following formulas:

$$
\begin{aligned}
& \overline{\hat{\alpha}}=\frac{1}{1000} \sum_{i=1}^{1000} \hat{\alpha}_{i}, \\
& \overline{\hat{\beta}}=\frac{1}{1000} \sum_{i=1}^{1000} \hat{\beta}_{i}, \\
& \operatorname{rmse} 1=\frac{1}{1000} \sum_{i=1}^{1000}\left[\left(\hat{\alpha}_{i}-\alpha\right)^{2}+\left(\hat{\beta}_{i}-\beta\right)^{2}\right]^{1 / 2},
\end{aligned}
$$




$$
\begin{aligned}
& \overline{\widetilde{\alpha}}=\frac{1}{1000} \sum_{i=1}^{1000} \tilde{\alpha}_{i}, \\
& \widetilde{\beta}=\frac{1}{1000} \sum_{i=1}^{1000} \tilde{\beta}_{i},
\end{aligned}
$$

and

$$
\text { rmse } 2=\frac{1}{1000} \sum_{i=1}^{1000}\left[\left(\tilde{\alpha}_{i}-\alpha\right)^{2}+\left(\tilde{\beta}_{i}-\beta\right)^{2}\right]^{1 / 2} \text {. }
$$

All simulation runs are performed by using a PC Pentium 4 with Matlab® 7.0.4 code. In this paper, we simulate three types of Weibull distribution shown in Table 1, in which one is decreasing failure rate, one is constant failure rate, and one is increasing failure rate. The sample means and the sample root mean square errors of $(\hat{\alpha}, \hat{\beta})$ and $(\tilde{\alpha}, \tilde{\beta})$ are calculated, respectively, for the three simulated Weibull distributions. All simulation results are recorded on Table 2. We assess performance of the maximum likelihood estimates and the least squares estimates by virtue of their individual sample root mean square errors. Examining all simulation results, we find that both $(\hat{\alpha}, \hat{\beta})$ and $(\tilde{\alpha}, \tilde{\beta})$ are more accurate with sample size $n$; that is, the sample root mean square errors of $(\hat{\alpha}, \hat{\beta})$ and $(\tilde{\alpha}, \tilde{\beta})$ are decreasing with sample size $n$. Table 2 implies that (i) if Weibull distribution holds decreasing rate function (e.g. $(\alpha, \beta)=(1,0.5)),(\tilde{\alpha}, \tilde{\beta})$ have smaller sample root mean square errors than $(\hat{\alpha}, \hat{\beta})$; (ii) if Weibull distribution holds constant rate function $($ e.g. $(\alpha, \beta)=(1,1))$, $(\tilde{\alpha}, \tilde{\beta})$ have smaller sample root mean square errors than $(\hat{\alpha}, \hat{\beta})$ when the sample size $n$ is small enough (e.g. $n \leq 40)$; and (iii) if Weibull distribution holds increasing rate function $($ e.g. $(\alpha, \beta)=(1,2)),(\hat{\alpha}, \hat{\beta})$ have smaller sample root mean square errors than $(\tilde{\alpha}, \tilde{\beta})$ when the sample size $n$ is large enough (e.g. $n \geq 20)$. In other words, the least squares method outperforms the maximum likelihood method when the underlying Weibull distribution has decreasing rate or constant rate with small sample size n; however, the maximum likelihood method dominates the least squares method if the underlying Weibull distribution has increasing rate with sufficiently large sample size $n$. 
Table 2. Sample means and sample root mean square errors of $(\hat{\alpha}, \hat{\beta})$ and $(\tilde{\alpha}, \tilde{\beta})$ for the three simulated Weibull distributions.

\begin{tabular}{|c|c|c|c|c|c|}
\hline $\begin{array}{l}\text { Parameter } \\
(\alpha, \beta)\end{array}$ & $\begin{array}{l}\text { Sample } \\
\text { size } n\end{array}$ & $\begin{array}{l}\text { Sample mean } \\
\text { of }(\hat{\alpha}, \hat{\beta})\end{array}$ & $\begin{array}{l}\text { Sample mean } \\
\text { of }(\tilde{\alpha}, \widetilde{\beta})\end{array}$ & $\begin{array}{l}\text { Sample rmse } \\
\text { of }(\hat{\alpha}, \hat{\beta})\end{array}$ & $\begin{array}{l}\text { Sample rmse } \\
\text { of }(\tilde{\alpha}, \tilde{\beta})\end{array}$ \\
\hline \multirow[t]{12}{*}{$(1.0,0.5)$} & 5 & $(2.11,0.71)$ & $(1.16,0.44)$ & 12.78 & $1.87^{*}$ \\
\hline & 10 & $(1.11,0.57)$ & $(0.99,0.43)$ & 0.55 & $0.36^{*}$ \\
\hline & 15 & $(1.06,0.54)$ & $(0.99,0.44)$ & 0.36 & $0.28^{*}$ \\
\hline & 20 & $(1.04,0.53)$ & $(0.99,0.44)$ & 0.30 & $0.25^{*}$ \\
\hline & 30 & $(1.01,0.52)$ & $(0.98,0.46)$ & 0.21 & $0.20^{*}$ \\
\hline & 40 & $(1.02,0.51)$ & $(0.98,0.46)$ & 0.19 & $0.17^{*}$ \\
\hline & 50 & $(1.01,0.51)$ & $(0.98,0.46)$ & $0.16^{*}$ & $0.16^{*}$ \\
\hline & 60 & $(1.01,0.51)$ & $(0.99,0.46)$ & 0.15 & $0.14^{*}$ \\
\hline & 70 & $(1.01,0.51)$ & $(0.99,0.47)$ & 0.14 & $0.13^{*}$ \\
\hline & 80 & $(1.01,0.50)$ & $(0.98,0.47)$ & $0.13^{*}$ & $0.13^{*}$ \\
\hline & 90 & $(1.01,0.50)$ & $(0.99,0.47)$ & $0.12^{*}$ & $0.12^{*}$ \\
\hline & 100 & $(1.00,0.50)$ & $(0.98,0.47)$ & $0.11^{*}$ & $0.11^{*}$ \\
\hline \multirow[t]{12}{*}{$(1.0,1.0)$} & 5 & $(1.72,1.47)$ & $(1.09,0.92)$ & 8.54 & $1.86^{*}$ \\
\hline & 10 & $(1.10,1.78)$ & $(0.99,0.87)$ & 0.61 & $0.44^{*}$ \\
\hline & 15 & $(1.08,1.11)$ & $(1.00,0.89)$ & 0.49 & $0.39^{*}$ \\
\hline & 20 & $(1.03,1.07)$ & $(0.98,0.90)$ & 0.35 & $0.31^{*}$ \\
\hline & 30 & $(1.04,1.05)$ & $(1.00,0.91)$ & 0.28 & $0.27^{*}$ \\
\hline & 40 & $(1.02,1.04)$ & $(0.99,0.93)$ & $0.23^{*}$ & $0.23^{*}$ \\
\hline & 50 & $(1.00,1.03)$ & $(0.98,0.93)$ & $0.20^{*}$ & 0.21 \\
\hline & 60 & $(1.00,1.02)$ & $(0.98,0.94)$ & $0.18^{*}$ & 0.19 \\
\hline & 70 & $(1.00,1.02)$ & $(0.98,0.94)$ & $0.16^{*}$ & 0.18 \\
\hline & 80 & $(1.00,1.01)$ & $(0.99,0.95)$ & $0.15^{*}$ & 0.16 \\
\hline & 90 & $(1.00,1.01)$ & $(0.99,0.95)$ & $0.14^{*}$ & 0.16 \\
\hline & 100 & $(1.00,1.01)$ & $(0.99,0.95)$ & $0.13^{*}$ & 0.15 \\
\hline \multirow[t]{6}{*}{$(1.0,2.0)$} & 5 & $(2.12,2.91)$ & $(1.12,1.81)$ & 19.51 & $1.93^{*}$ \\
\hline & 10 & $(1.10,2.32)$ & $(0.99,1.73)$ & 0.90 & $0.71^{*}$ \\
\hline & 15 & $(1.05,2.19)$ & $(0.98,1.75)$ & 0.62 & $0.57^{*}$ \\
\hline & 20 & $(1.04,2.14)$ & $(0.98,1.77)$ & $0.51^{*}$ & $0.51^{*}$ \\
\hline & 30 & $(1.03,2.08)$ & $(0.99,1.81)$ & $0.39^{*}$ & 0.42 \\
\hline & 40 & $(1.01,2.06)$ & $(0.98,1.83)$ & $0.31^{*}$ & 0.37 \\
\hline
\end{tabular}




\begin{tabular}{|l|l|l|l|l|l|}
\hline & 50 & $(1.02,2.06)$ & $(0.99,1.86)$ & $0.29^{*}$ & 0.34 \\
\cline { 2 - 6 } & 60 & $(1.01,2.05)$ & $(0.98,1.88)$ & $0.26^{*}$ & 0.32 \\
\cline { 2 - 6 } & 70 & $(1.00,2.03)$ & $(0.99,1.88)$ & $0.23^{*}$ & 0.29 \\
\hline & 80 & $(1.01,2.04)$ & $(0.99,1.90)$ & $0.21^{*}$ & 0.26 \\
\hline & 90 & $(1.00,2.02)$ & $(0.99,1.90)$ & $0.20^{*}$ & 0.26 \\
\hline & 100 & $(1.00,2.02)$ & $(0.99,1.90)$ & $0.19^{*}$ & 0.24 \\
\hline
\end{tabular}

*denotes the smaller sample root mean square errors of $(\hat{\alpha}, \hat{\beta})$ and $(\tilde{\alpha}, \tilde{\beta})$ for the three simulated Weibull distributions.

\section{CONCLUSIONS}

This paper explores behavior of the maximum likelihood method and the least squares method used to estimate Weibull parameters $(\alpha, \beta)$. Through a numerical simulation study, we realize performance of these two estimation method. Based upon the criterion of sample root mean square errors, we found that the least squares method is superior to the maximum likelihood method when the sample size is small enough. Alternatively, the maximum likelihood method performs better if the sample size is large enough. We summarize the above results in the following Table 3.

Table 3. Comparison of the maximum likelihood method and the least squares method for estimating Weibull parameter $(\alpha, \beta)$

\begin{tabular}{|c|c|c|c|}
\hline \multirow{2}{*}{$\begin{array}{c}\text { Level of shape } \\
\text { parameter }\end{array}$} & Type of rate function & $\begin{array}{c}\text { Range of } \\
\text { sample size }\end{array}$ & Better method \\
\hline \multirow{2}{*}{$0<\beta<1$} & Decreasing rate & Any size & The least squares \\
\hline \multirow{2}{*}{$\beta=1$} & Constant rate & $n \leq 40$ & The least squares \\
\cline { 3 - 4 } & \multirow{2}{*}{$\beta>1$} & $n>40$ & The maximum likelihood \\
\hline \multirow{2}{*}{ Increasing rate } & $n \leq 20$ & The least squares \\
\cline { 3 - 4 } & & $n>20$ & The maximum likelihood \\
\hline
\end{tabular}

In addition, Table 2 also indicates that the estimates $(\hat{\alpha}, \hat{\beta})$ have significantly larger sample root mean square errors than $(\tilde{\alpha}, \tilde{\beta})$ when the sample size $n \leq 15$. This 
may be due to the fact that the maximum likelihood estimating equations (equations (7) and (8)) have no regularity condition for determining the existence of the solution - the maximum likelihood estimates $(\hat{\alpha}, \hat{\beta})$. Apparently, the least squares method doesn't have this shortcoming because the least squares estimates $(\tilde{\alpha}, \tilde{\beta})$ can be calculated explicitly by equations (14), (15), and (17). Note that using the least squares method to estimate the Weibull parameters $(\alpha, \beta)$ can overcome the shortcoming caused by the maximum likelihood method when the sample size is not sufficiently large. Further research may consider the investigations of interval estimation and hypothesis testing for Weibull parameters $(\alpha, \beta)$ by means of the least squares method.

\section{REFERENCES}

1. A. Hoyland and M. Rausand, System Reliability Theory-Models and Statistical Methods, John Wiley \& Sons, Inc., 1994.

2. M. Yang and H. Nie, Advanced Algorithm for Maximum Likelihood Estimation of Three Parameter Weibull Distribution, Journal of Nanjing University of Aeronautics \& Astronautics, 01, 2007.

3. J. Dai, A. Yang, and Z. Yang, An Advanced Algorithm for Equations of Maximum Likelihood Estimation, Applied Mathematics A Journal of Chinese Universities (Ser. A), 03, 2009.

4. J. Shi, X. Ren, X. Chen, and X. Li, A Solution Method for 3-Parameter Weibull Distribution of Maximum Likelihood Estimation, Henan Science, 07, 2009.

5. L. Wasserman, All of Statistics- A Concise Course in Statistical Inference, Springer Science+Business Media, Inc., 2004.

6. R.V. Hogg, J.W. Mckean, and A. T. Craig, Introduction to Mathematical Statistics, Sixth Edition, Pearson Education, Inc., 2005.

7. G. G. Roussas, A Course in Mathematical Statistics, Second Edition, Academic Press, 1997. 